\title{
Die Anwendung des Phasenkontrast-Verfahrens zum Studium der Pflanzenzelle
}

\author{
Von Siegrried Strugger \\ Aus dəm Botanischen Institut der Tierärztlichen und Technischen Hochschule Hannover \\ (Z. Naturforschg. 2 b, 146-152 [1947]; eingegangen am 17. Januar 1947)
}

\begin{abstract}
Das von Zernike ${ }^{1}$, Köhler und Loos ${ }^{2}$ konstruierte Phasenkontrast-Mikroskop wird erstmalig an lebenden Metaphytenzellen zu zytologischen Untersuchungen angewandt. Zur Beobachtung der Plasmakonfiguration, der Chondriosomen sowie zur Analyse der Zytoplasmaströmung ist dieses neue Mikroskopierverfahren bestens geeignet. Modellversuche zeigten, daß insbesondere nucleinsäure- und lipoidreiche Strukturen den stärksten Phasenkontrast liefern. Daher ist das Phasenkontrast-Verfahren für die Untersuchung der Struktur lebender Ruhekerne von besonderer Bedeutung. Die Chromomeren geben einen sehr starken Phasenkontrast. An isolierten Chloroplasten gelang es, durch die stark aufquellende Wirkung von KCNS die Proteinlamellen der Chloroplasten so zu verbreitern, daß der lamellare Feinbau der Chloroplasten im Phasenkontrastbild mikroskopisch sichtbar gemacht werden kann. Die wie ein Buch aufgeblätterten Chloroplasten lassen dann hell erscheinende Proteinschichten und dunkel kontrastierte Lipoidschichten erkennen. Der lamellare Feinbau konnte an Stichproben aus verschiedenen Angiospermenfamilien allgemein bestätigt werden. Die autochthone Stärke liegt in den hell erscheinenden quellfähigen Proteinschichten. Für das Studium der Kern- und Zellteilung in vivo an den Staubfadenhaaren von Tradescantia virginica hat sich das Phasenkontrast-Mikroskop als sehr geeignet erwiesen. Es werden einige Einzelheiten über die Chromosomen, die Spindel, den Phragmoplasten und über die Entstehung der Quermembran mitgeteilt.
\end{abstract}

A. Köhler und W. Loos haben 1941, aufbavend auf den von Zernike ${ }^{1}$ geschaffenen physikalischen Grundlagen, das Phasenkontrast-Verfahren als neue mikroskopische Untersuchungstechnik eingeführt. Michel $l^{3}$ konnte zeigen, daß mit Hilfe des Phasenkontrast-Mikroskopes die Spermatozyten von Heuschrecken und die Speicheldrüsenzellen der Chironomiden in vivo besonders gut zu. brobachten sind. Die Chondriosomen und die Chromosomen geben am ungefärbten lebendigen Präparat einen starken Phasenkontrast. Ilse Fischer und Michel haben im Laboratorium der Zeiß-Werke (Jena) mit Erfolg die Kernund Zellteilung an Heuschrecken-Spermatozyten im Zeitrafferfilm festhalten können.

Auf dem Gebiet der Mikrobiologie liegt der erste Bericht über die Anwendung des PhasenkontrastMikroskopes von H. Knölli ${ }^{4}$ vor. Lebende Bakterienzellen geben einen guten Phasenkontrast, so daß Bakterien fast so kontrastreich in vivo abbildbar sind wie im gefärbten Ausstrichpräparat. Auch kernähnliche Körper konnte Knöll in Bakterienzellen mit Hilfe des Phasenkontrast-Mikroskopes beobachten. Als besonders vorteilhaft hat sich das

1 F. Zernike, Monthly Notices Roy. astronom. Soc. 94, 377 [1934]; Physica 1, 689 [1934]; Z. techn. Physik 16, 454 [1935].
Phasenkontrast-Verfahren zur mikroskopischenVerfolgung von Bakterienmikrokulturen erwiesen.

Über die Brauchbarkeit des PhasenkontrastMikroskopes zum Studium der lebenden Metaphytenzelle liegt bisher in der Literatur keine Angaba vor. Die in dieser Arbeit mitgeteilten Beobachtungsergebnisse stellen somit den ersten Versuch dar, das Phasenkontrast-Verfahren zur Analyse der Metaphytenzelle heranzuziehen.

Es gibt Amplituden- und Phasenpräparate. Ein Amplitudenpräparat ist z. B. ein gefärbtes Schnittpräparat. Hier erfolgt die Kontrastierung der Strukturen durch wechselnde Lichtabsorption. Es ändert sich also im wesentlichen die Amplitude der Wellenbewegung des Lichtes beim Durchgang durch solche Präparate. Die klassische Zytologie hat ihre Beobachtungstatsachen hauptsächlich an solchen Amplitudenpräparaten gewonnen. Lebende Zellen und Gewebe sind dagegen meist farblos. In den wasserreichen Gel- und Solstrukturen des lebenden Zellinhaltes werden die Amplituden der durchgehenden Lichtwellenbewegung nicht geändert, wohl aber infolge der verschiedenen Brechungsindices der Strukturen Phasenänderungen (Gangunterschiede) erzeugt. Phasenänderungen ergeben aber im Hellfeldmikroskop nur sehr geringfügige Kontraste, welche durch Zuziehen der Blende auf Kosten eines Aperturverlustes etwas gesteigert werden können. Daher werden Chondriosomen und Chromosomen sowie Plasma-

2 A. Köhler u. W. Loos, Naturwiss. 29, 49 [1941].

3 K. Michel, Naturwiss. 29, 61 [1941].

4 H. Knöll. Zeiß-Nachr. 5. Folge, H. 2, 38 [1944]. 
stränge u. a. am lebenden Material im ungefärbten Zustand selbst bei Anwendung der besten Optik nur sehr flau und undeutlich abgebildet. Lebende Zellen und Gewebe sind sonach ausgesprochene Phasenpräparate. Auf dieser Tatsache beruht die Schwierigkeit der mikroskopischen Lebendbeobachtung der Zellen und ihrer Strukturen. Mit Hilfe der von Köhler und Loos ${ }^{2}$ eingeführten Phasenkontrast-Optik können Phasen- in Amplitudenänderungen umgewandelt werden. Phasenpräparate werden mit Hilfe des Phasenkontrast-Mikroskopes so abgebildet, als ob sie ursprünglich Amplitudenpräparate wären. Für die zytologische Analyse lebender Metaphytenzellen ist sonach das Phasenkontrast-Verfahren vom theoretischen Gesichtspunkt aus wohl die geeignetste Untersuchungsmethodik. Unter der Voraussetzung, daß das Phasenkontrast-Mikroskop am pflanzlichen Zellmaterial überhaupt anwendbar ist, kann die Prognose gestellt werden, da B das Phasenkontrast-Verfahren dazu berufen ist, eine empfindliche Lücke in der optischen Methodik der Lebendbeobachtung von Zellen und Geweben in Zukunft auszufüllen.

I. Untersuchungen an den oberen Epidermiszellen der Zwiebelschuppe von

$$
\text { Allium Cepa }
$$

Fürdie Bildung eines Urteils über die Brauchbarkeit des Phasenkontrast-Mikroskopes schien mir dieses zytologisch wohlbekannte Objekt zunächst am geeignetsten zu sein. Die Präparation und Isolierung der Epidermis habe ich in der von $\operatorname{mir}^{\mathbf{5}}$ beschriebenen Art und Weise vorgenommen. Die Einbettung der Epidermen erfolgte in Leitungswasser. Es müssen extra dünne Deckgläser verwendet werden, wobei darauf zu achten ist, daß die Flüssigkeitsschicht zwischen Objektträger und Deckglas möglichst dünn sein soll. Eine Quetschung des Epidermisgewebes mv $B$ jedoch vermieden werden. Es wurde mit der von Zeiß (Jena) gelieferten Phasenkontrast-Einrichtung gearbejtet. Als Lichtquelle diente eine Mikroskopier-Niedervoltlampe Lux FNI von Reichert (Wien). Alle Beobachtungen sind mit positivem Phasenkontrast gemacht worden. Die Untersuchung der Zellen wurde mit den Phasenkontrast-Objektiven $\mathrm{Ph} \mathrm{20,}$ $\mathrm{Ph} 40$ und der homogenen Immersion Ph 90 vorgenommen. Parallel zur Phasenkontrast-Untersuchung wurde das entsprechende Hellfeldbild ständig vergleichsweise betrachtet.

Bei schwacher Vergrößerung (Ph 20) sind Einzelheiten in den Zellen im Phasenkontrastbild nicht wesentlich besser zu beurteilen als im Hellfeld. Wohl aber heben sich die Protoplasten in dunklem Kontrast hervor, so daß die Plasmakonfiguration

5 S. Strugger, Praktikum der Zell- und Gewebephysiologie der Pflanze. Verlag Gebr. Bornträger, Berlin 1935. viel klarer zu beobachten ist als im normalen Hellfeld. Plasmolysebilder sind daher im Phasenkontrast-Mikroskop bei schwacher Vergrößerung besonders schön zu beobachten und können wesentlich leichter mikrophotographisch festgehalten werden. Sehr deutlich sind die Kerne kontrastiert. Wird das Objektiv $\mathrm{Ph} 40$ eingestellt, so fällt die günstige Kontrastwirkung schon mehr ins Auge. Die Chondriosomen, die Mikrosomen und die Kernstrukturen treten scharf hervor. Noch besser ist aber die Kontrastwirkung bei der Verwendung der homogenen Immersion Ph 90. Das PhasenkontrastVerfahren ist dann dem normalen Hellfeldmikroskop so überlegen, daß über die Bedeutung der Phasenkontrast-Mikroskopie für die zukünftige Zellforschung kein Zweifel mehr bestehen kann. Es sollen daher einige wesentliche Einzelheiten mitgeteilt werden.

Die Zellmembran tritt im Phasenkontrastbild sehr stark lichtbrechend hervor. Helle Diffraktionsräume begleiten die Antiklinenmembranen. Die Flächenmembranen (Periklinen) stören dagegen das Phasenkontrastbild überhaupt nicht, so daß meine ursprünglichen Befürchtungen bezüglich des störenden Einflusses der Zellmembranen sich nicht bewahrheitet haben.

Die Zytoplasma-Grundsubstanz ist als homogen grau erscheinende Masse hervorgehoben. Das Zytoplasma sieht so aus, als ob es intravital schwach grau gefärbt wäre. Ich konnte keinerlei Struktur in der Zytoplasma-Grundsubstanz beobachten. Die Plasmafäden und Plasmastränge sind besonders hervorgehoben. Alle Grenzflächen zwischen Plasma und Zellsaftraum sind als scharfe schwarze Linien zu beobachten.

Besonders deutlich sind die Zytoplasma-Einschlüsse hervorgehoben. Die im Hell- und Dunkelfeld stark hervortretenden Mikrosomen erscheinen schwarz konturiert mit einem hell leuchtenden Innern. Sie sind im Phasenkontrastbild sehr leicht von den Chondriosomen zu unterscheiden. Während die Chondriosomen im Hell- und Dunkelfeld nur sehr schwer zu beobachten sind, treten sie im Phasenkontrast-Mikroskop als homogene, schwarzgrau getönte Gebiłle deutlich in Erscheinung. Sie zeigen in ihrem Innern keine Diffraktionserscheinungen. Runde, ovale, stäbchen- und hantelförmige Chondriosomen sind so schön zu sehen, als ob sie vital gefärbt wären. Es besteht kein Zweifel, daß für zukünftige experimentell-zytologicche Untersuchungen an Chondriosomen das Phasenkontrast-Mikro- 
skop ein unentbehrliches Rüstzeug sein wird. Die Leukoplasten verhalten sich gleich wie die Chondriosomen. Sie erscheinen im Phasenkontrastbild homogen dunkel kontrastiert und zeichnen sich lediglich durch ihre Größe aus. Runde, hantelförmige, geweihartig verzweigte oder spindelförmig ausgezogene Formen konnten beobachtet werden. Aus diesem Befund kann geschlossen werden, daß am geeigneten Material das Phasenkontrast-Mikroskop für die weitere Erforschung der Proplastiden in $\mathrm{Zu}$ kunft von Bedeutung sein wird.

Der Zellkern hebt sich schon bei schwacher Vergrößerung dunkel hervor. Er ist in der lebenden Zelle so zu beobachten, als ob er gefärbt wäre. Mit dem Immersionsobjektiv Ph 90 betrachtet, enthüllt sich dem Beobachter ein eindrucksvolles Bild seiner Struktur. Die Kernmembran ist als schwarz erscheinende Kontur zu erkennen. Dort, wo die Oberfläche des flachen, scheibenförmigen Kernes mit Rillen versehen ist, kann an den Rillenkanten die Kernmembran besonders gut beobachtet werden. Die Nucleolen geben einen fast schwarzen Phasenkontrast. Für die zytologische Forschung ist der durch die Phasenkontrast-Optik gewährte Einblick in die Struktur des ruhenden Kernes von größtem Interesse. Das ganze Kerninnere ist mit regelmäßigen, in linearen Reihen angeordneten, feinen, schwarz erscheinenden Körnchen angefüllt, so daß der Eindruck eines regelmäßig gebauten, feinen Raumgitters entsteht, von dem nur die dunkel kontrastierten Körnchen zu sehen sind. Da schon Mich e $\mathbf{l}^{\mathbf{3}}$ zeigen konnte, daß die thymonucleinsävrehaltigen Chromomeren den stärksten Phasenkontrast liefern, liegt es nahe, die Annahme zu. machen, daß die den Kern erfüllenden, linear angeordneten Körnchen mit den Chromomeren identisch sind. Mit keiner optischen Methode ist die Struktur des Ruhekernes von Allium Cepa im lebenden Zustand so klar aufzulösen wie mit dem Phasenkontrast-Mikroskop. Die Abb. 1, 2 und 3 (s.Tafel, S. 136 b) geben Bilder von der Struktur des Ruhekernes von Allium-Zellen, gewonnen im Hellfeld, D unkelfeld und Phasenkontrast-Mikroskop bei gleicher Vergrößerung. Im Hellfeldbild ist die Chromomerenstruktur mit bester Objektivoptik - Zeiß Apochromat 60, n.A. 1,4 - nur andeutungsweise zu erkennen. Über die Form und Größe der Chromomeren kann das Hellfeld keine sichere Auskunft geben. Im Dankelfeld kommen zwar die Chromomeren als silbrig leuchtende Gebilde klarer hervor, doch geben die Beugungsbilder keinen exakten Einblick in die wahre Struktur des Ruhekernes. Im Phasenkontrastbild werden die Chromomeren in voller Klarheit abrebildet.

Für weitere Forschungsarbeiten auf dem Gebiete der Kernstrukturforschung am lebenden Material wird also das Phasenkontrast-Verfahren in Zukunft die Methode der Wahl sein.

Die überaus klare Kontrastierung der Zytoplasmagrundsubstanz sowie der Mikrosomen und Chondriosomen ermöglicht es, den Verlauf der Zytoplasma-Strömung im Phasenkontrast-Mikroskop bis in die feinsten Einzelheiten hinein zu verfolgen. In der Allium-Zelle ist die Zytoplasmabewegung sehr fein differenziert. Streng lokalisierte Bezirke mit bestimmt gerichteten, häufig in ihrer Richtung wechselnden Bewegungen der Einschlüsse sowie ein ständiger Wechsel des Bewegungsausmaßes sind im Phasenkontrast-Mikroskop klar zu beobachten. Das gewonnene Bild fügt sich gut in die theoretische Vorstellung, daß ganze Eiweißmolekülkomplexe durch die freie Drehbarkeit der Polypeptidketten im Laufe der Stoffwechselprozesse sich kontrahieren und wieder ausdehnen. Zum experimentellen Studium der Zytoplasmaströmung kann die Anwendung der Phasenkontrast-Optik nur empfohlen werden.

An plasmolysierten Zellen liefert das Phasenkontrast-Mikroskop wertvolle Aufschlüsse. Die Hechtschen Fäden, intrazelluläre Myelinfiguren und Lipoidtropfen, sowie bei Tonoplastenplasmolysen die Tonoplasten liefern einen starken Phasenkontrast.

II. Orientierende Beobachtungen an einigen Modellen

Die Beobachtungen an den Epidermiszellen von Allium Cepa haben ergeben, daß innerhalb der Zelle der stärkste Phasenkontrast vom Chromatin und den Nucleolen geliefert wird. Ferner sind die lipoidführenden Strukturen wie die Chondriosomen, die Tonoplasten, die Kernmembran und intrazellvläre Myelinfiguren durch eine starke Kontrastierung ausgezeichnet. Das Zytoplasma, welches lipoidärmer und eiweißreicher ist, gibt nur einen schwachen Phasenkontrast.

Es war daher wichtig, Phasenkontrast-Untersuchungen an Modellen durchzuführen. Für das Chromatin wurde als Modell ein in Wasser fein dispergiertes Präparat von Nucleinsäure (aus Hefe), Merck, verwendet. Die Untersuchungen im Phasenkontrast-Mikroskop ergaben eine schwarze Kon- 
trastierung der mikroskopisch kleinen Nucleinsäureflocken. Dieses Verhalten stimmt somit gut mit der starken Kontrastierung der Chromomeren und Nucleolen überein.

Als Modell für lipoidreiche Strukturen zog ich künstlich erzeugte, mikroskopisch kleine Myelinfiguren heran. Ein kleiner Tropfen Ölsäure wird unter dem Deckglas mit konz. Ammoniak in Berührung gebracht. Die Untersuchung der an der Grenzfläche entstehenden Myelinfiguren mit der Phasenkontrast-Optik bestätigte die schon an der lebenden Zelle gemachte Erfahrung, daß lipoidreiche Gebilde und feinste Filme einen sehr starken Phasenkontrast liefern.

\section{Untersuchungen an Chloroplasten}

Aus Querschnitten durch Laubblätter von Allium Cepa wurden durch sorgfältiges Präparieren einzelne lebende Palisadenzellen isoliert. Eingebettet in Leitungswasser eigneten sie sich ausgezeichnet zur Phasenkontrast-Untersuchung lebender Chloroplasten. Diese geben einen starken Phasenkontrast (Lipoidreichtum!). Thre grüne Farbe ist weitgehend verschwunden und sie erscheinen dunkelgrau. Die Untersuchung mit der homogenen Immersion $\mathrm{Ph} 90$ läßt klar den Grana-Feinbau erkennen. Das Stroma erscheint hellgrav, während die Grana sich im dunkelgrauen Phasenkontrast abheben. Mechanisch geschädigte Palisadenzellen enthalten Chloroplasten, welche die Anzeichen aller bekannten Desorganisationsstadien erkennen lassen. Das Streifigwerden sowie Vakuolisationserscheinungen aller Art sind im Phasenkontrastbild deutlicher zu beobachten als im Hellfeld.

$\mathrm{Da}$ die Chloroplasten in den Zellen meist sehr dicht gelagert sind, bin ich dazu übergegangen, Chloroplasten zu isolieren und sie in verschiedenen Medien unter dem Phasenkontrast-Mikroskop zu beobachten. Wird das Mesophyll durch Anschaben verletzt, so lassen sich aus den Blättern der verschiedensten Pflanzen Chloroplasten bequem isolieren. Je nach der Natur des Mediums, in welches sie eingebettet werden, erleiden sie dann charakteristische degenerative Veränderungen.

Ausgehend von der durch Frey-Wyssling ${ }^{6}$ entwickelten Vorstellung, nach welcher der Chloroplast aus parallel zur Fläche gelagerten Eiweiß- und Lipoidlamellen in regelmäßiger Abwechslung auf-

- A. Frey-Wyssling, Submikroskopische Morphologie des Protoplasmas und seiner Derivate. Protoplasma Monographien 15. Bd. [1935]. gebaut ist, deren Dimensionen submikroskopisch sind, und ausgehend von der Erfahrung, daß selbst feinste Lipoidlamellen, in der Seitenansicht beobachtet, einen sehr starken Phasenkontrast liefern, habe ich die isolierten Chloroplasten in Lösungen von KCNS eingebettet und im Phasenkontrast-Mikroskop bei stärkster Vergrößerung beobachtet. KCNS ist ein sehr starkes Quellungsmittel für Eiweißgele. Sollte es gelingen, die Eiweißlamellen so stark durch maximale, künstlich hervorgerufene Quellung zu verdicken, daß sie mikroskopische Dimensionen annehmen, so müssen in der Kantenansicht der Chloroplasten die dunkel kontrastierten Lipoidlamellen in Abwechslung mit den gequollenen, heller erscheinenden Eiweißlamellen mikroskopisch sichtbar werden. Das Ziel der Untersuchungen an isolierten Chloroplasten war, die hypothetisch erschlossene, durch polarisationsoptische Untersuchungen von Menke $\mathrm{e}^{7}$ wahrscheinlich gemachte submikroskopicche Lamellenstruktur der Chloroplasten durch diesen technischen Kunstgriff mikroskopisch nach zuweisen.

Die entsprechenden Versuche zeigten in der Tat die Richtigkeit der obigen Überlegung. Werden Chloroplasten von Allium-Cepa-Blättern im isolierten Zustand in 0,6-1-m. KCNS-Lösungen eingebettet und im Phasenkontrast-Mikroskop untersucht, so sind folgende Veränderungen an den in der Seitenansicht liegenden Chloroplasten zu beobachten:

Allmählich nimmt zunächst die Dicke der linsenförmigen Chloroplasten zu. Diese Aufquellung schreitet ständig weiter, und gleichzeitig wird eine feine, streifige Struktur parallel zur Linsenfläche der Chloroplasten sichtbar. Feine, schwarz erscheinende Lamellen sind in paralleler Lagerung im Chloroplasten zu beobachten. Zwischen diesen befinden sich hell erscheinende Zwicchenräume. Unter ständiger Aufblätterung des Chloroplasten vergrößern sich die helleren Zwischenräume und die Lamellen bauchen sich schließlich nach beiden Seiten halbkugelig au.s. Man hat den Eindruck, als ob der Chloroplast wie ein Buch aufgeblättert wird. Schließlich stellt der ganze Chloroplast ein aufgeblättertes Lamellenmagazin dar. Abb. 4 zeigt einen Chloroplasten von Allium Cepa bei Beginn der Quellung (a) und am Ende der Quellung (b), in 0,6m. KCNS. Im Durchschnitt kann man in den aufgeblätterten Chloroplasten etwa 25 dunkle (Li-

7 W. Menke, Kolloid-Z. 85, 256 [1938]. 


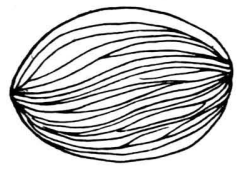

a

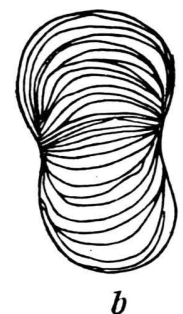

$\boldsymbol{b}$
Abb. 4. Isolierte Chloroplasten aus dem Laubblattmesophyll von Allium Cepa, eingebettet in 0,6-m. KCNS. Seitenansicht. a) Beginn der Aufquellung und Aufblätterung, b) maximale Quellung und Aufblätterung. Die Lipoidlamellen erscheinen dunkel, während die Proteinschichten hell erscheinen.

poid-)Lamellen als Rechengrundlage annehmen. Zur Vereinfachung kann fernerhin angenommen werden, daß die Lipoid- und Proteinlamellen etwa gleich dick sind (was in Wirklichkeit aber theoretisch unwahrscheinlich ist). Ein Chloroplast wäre dann aus 25 Lipoid- und 25 Proteinlamellen im Durchschnitt aufgebaut. Unter der Voraussetzung, daß der Chloroplast $3 \mu$ dick ist, ist im lebenden Chloroplasten 1 Lamelle 600 Å-Einheiten dick oder $0,06 \mu$. Diese Lamellen sind also sicher im normalen Chloroplasten submikroskopisch dimensioniert. Auf Grund dieser Überschlagsberechnung kann also mit Recht die Annahme gemacht werden, daß die in KCNS sichtbaren Lamellensysteme tatsächlich den submikroskopisch dimensionierten Lamellensystemen des ungeschädigten Chloroplasten entsprechen. Es ist sogar wahrscheinlich, daß alle oder fast alle Proteinschichten aufgequollen sind. Nähere quantitative Untersuchungen über dieses Problem sind in einer weiteren Untersuchungsreihe geplant.

Es besteht sonach kein Zweifel, daß der submikroskopische Feinbau der Chloroplasten durch die starke Aufquellung der Proteinschichten sich im Phasenkontrastbild durch direkte mikroskopische Beobachtung belegen läßt. An dem lamellaren Feinbau, bestehend aus Protein- und Lipoidschichten, kann nach diesen Beobachtungen kein Zweifel mehr bestehen.

Es war zunächst die Frage zu klären, ob sich der lamellare Feinbau mit Hilfe der oben angegebenen Methodik auch an Chloroplasten von anderen Pflanzen nachweisen läßt. Diese Frage ist insofern bedeutungsvoll, als sich durch diese Untersuchungen die Frage entscheiden läßt, ob die Chloroplasten aller Angiospermen einen einheitlichen Feinbau besitzen. Ich konnte an Stichproben aus verschiedenen Angiospermenfamilien ganz allgemein den lamellaren Feinbau der Chloroplasten bestätigt finden.
Allium Cepa gehört zu den saccharophyllen Pflanzen. Als Vertreter der amylophyllen Pflanzen sei noch Atriplex hastata kurz besprochen. Die isolierten Chloroplasten dieser Pflanze geben in 0,6$m$. KCNS eine besonders schöne Aufblätterung. Chloroplasten, welche vor dem experimentellen Eingriff durch Assimilation autochthone Stärke gebildet hatten, ließen nach erfolgter Aufquellung im Phasenkontrast-Mikroskop die Lokalisation der autochthonen Stärke klar erkennen. Wie Abb. 5 zeigt, wird die autochthone Stärke in den Proteinschichten und nicht in den Lipoidschichten gebildet.

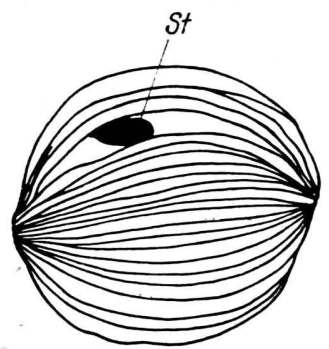

Abb. 5. Isolierter Chloroplast aus dem Blattmesophyll von Atriplex hastata, eingelegt in 0,6-m. KCNS. Seitenansicht. Starke Aufquellung und Aufblätterung der submikroskopischen Schichtung. St : autochthones Stärkekorn, welches deutlich in die quellfähige Proteinschicht eingelagert ist.

IV. Bemerkungen zur Beobachtung der Kern- und Zellteilung an den Staubfadenhaaren von Tradescantia virginica

Dieses klassische Objekt habe ich zur Prüfung der Frage herangezogen, ob das Phasenkontrast-Verfahren in gleicher Weise wie am tierischen Objekt auch am pflanzlichen Material eine vorteilhafte Beobachtung des Ablaufs der Kern- und Zellteilung ermöglicht. Junge Staubfadenhaarzellen wvrden in 1-proz. Glucose-Lösung (Leitungswasser) unter Deckglasabschluß einer Daverbeobachtung im Phasenkontrast-Mikroskop unterworfen. Der Ablauf der Kern- und Zellteilung war dann bei günstigen Temperaturverhältnissen $\left(20-25^{\circ}\right)$ innerhalb eines Zeitraumes von $\mathbf{1}^{\mathbf{1} / \mathbf{2}}-2^{\mathbf{1}} / \mathbf{2}$ Stdn. zu. beobachten. Auf eine ausführliche Schilderung der Beobachtungen soll hier verzichtet werden. Ich möchte daher nur auf einige interessante Einzelbeobachtungen eingehen. Sowohl die Struktur des Ruhekernes als auch die Chromosomen sind in allen Stadien der Kernteilung durch einen starken Phasenkontrast so gut hervorgehoben, daß der gesamte Teilungsverlauf ohne besondere technische Schwierigkeiten in einem Filmstreifen festgehalten werden könnte. 
Im Ruhekern sind die Chromomeren in linearer Anordnung sehr klar zu beobachten. Auch die Nucleolen sind dunkel hervorgehoben. Die Kernmembran liegt an der Grenze der mikroskopischen S.chtbarkeit und ist gerade ncch zu. erkennen. - Dər Ablauf der Kernteilung entspricht in allen Einzelheiten den Bildern, welche Bělı r.8 (1929) an mit Osmiumsäure fixiertcm Material niedergelegt hat. Es bestehen zu dieser Darstellung im wesentlichen nur zwei Differenzen. 1. Während am fixierten und gefärbten Material die Chromosomen kompakt erscheinen, ist im Phasenkontrast-Mikroskop der Chromomeren-Feinbau der Chromosomen in allen Stadien der Kernteilung und auch im MetaphasenChromosom noch zi: sehen. 2. Die Spindelfasern konnten im Phasenkontrast niemals festgestellt werden. Ich konnte auch keine Inhomogenität der Spindelrävme beobachten, welche mit den Spindelfasern in Beziehung gebracht werden könnte.

Das Phasenkontrast-Verfahren gab fernerhin einen klaren Aufschlvß über die Vorgänge bei der Bildung der Quermembran in der beginnenden Telophase. Zunächst bildet sich offensichtlich aus dem Zytoplasma der homogen hell erscheinende Phragmoplast. Innerhalb weniger Sekunden treten dann im Phragmoplasten kleine, schwarz erscheinende Körnchen (Tröpfchen) auf, welche in reger Brownscher Molekularbewegung tanzen. In wenigen Sekunden ordnen sich, dann diese Tröpfchen, von der Zellmitte ausgehend, zu einer äquatorialen Platte an, welche aus einer Tröpfchenreihe gebildet wird.

8 K. Běla ř, Z. Zellforschg. mikroskop. Anat. 10, 73 [1929].

9 W.A. Becker, Protoplasma 15, 478 [1932]; Acta soc. bot. polon. 9, 381 [1932]; C. R. hebd. Séances Acad. Sci. 196, ¿022 [1933]; Acta. soc. bot. polon. 11, 139 [1934]; Z.
Im Verlauf von weiteren 30 Sek. fließen dann diese Tröpfchen zu. einer dunkel kontrastierten Lamelle zusammen, welche bald ein vollständiges Querseptum darstellt. Der homogene Phragmoplast wird allmählich zurückgebildet und normales Zytoplasma tritt an seine Stelle. Vergleicht man die von Běla $\check{r}^{8}$ in Tafel III, Fig. 16, 17, 18, wiedergegebenen Mikroaufnahmen mit dem soeben geschilderten Phasenkontrastbefund, so wird erst der große cptische Fortschritt klar, der durch das Phasenkontrast-Verfahren gegeben ist. Die Bildung der ersten Anlage der Quermembran, wie sie im Phasenkontrastbild zu beobachten ist, deckt sich weitgehend mit den Beobachtungen von Becker ${ }^{9}$ und Ellenhorn ${ }^{\mathbf{1 0}}$. Beide Autoren beschreiben das Auftreten von Tröpfchen im Phragmoplasten vind deren Beteiligung an der Bildung der neuen Quermembran. Es ist von besonderem Interesse, daß Becker in seinen ausgezeichneten Arbeiten über die Vitalfärbung der sich teilenden Zellen mit Azofarbstoffen zu der Überzeugung gelangte, daß diese Tröpfchen lipoider Natur sind, was mit dem Verhalten derselben im Phasenkontrast-Mikroskop sehr gut übereinstimmt.

Besonders schön sind im Telophasenkern die Chromosomenareale und die allmähliche Auflockerung (Aufrollung) der Chromonemata zu beobachten.

Schon aus diesen kurzen Mitteilungen geht hervor, daß das Phasenkontrast-Verfahren zur Klärung zytologischer Fragen am lebenden Objekt in $\mathrm{Zr}$ :kunft eine bedeutende Rolle spielen wird.

Zellforschg. mikroskop. Ar.at. 23, 253 [1935]; Cytologia 6, 337 [1935].

10 J. Ellenhorn, Z. Zellforschg. mikroskop. Anat. 20, 288 [1933]. 